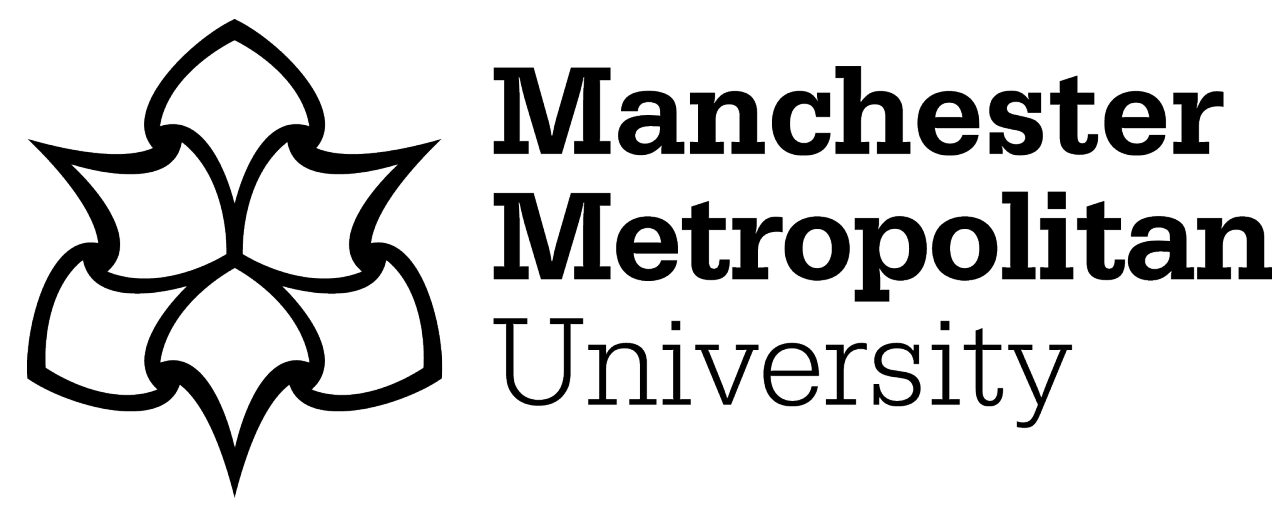

Moretti, A ORCID logoORCID: https://orcid.org/0000-0001-6543-9418, Shlomo, N and Sakshaug, JW (2020) Multivariate Small Area Estimation of Multidimensional Latent Economic Well-being Indicators. International Statistical Review, 88 (1). pp. 1-28. ISSN 0306-7734

Downloaded from: https://e-space.mmu.ac.uk/625180/

Version: Accepted Version

Publisher: Wiley

DOI: https://doi.org/10.1111/insr.12333

Please cite the published version 


\title{
Multivariate Small Area Estimation of Multidimensional Latent Economic Wellbeing Indicators
}

\section{Manuscript}

\begin{abstract}
Factor analysis models are used in data dimensionality reduction problems where the variability among observed variables can be described through a smaller number of unobserved latent variables. This approach is often used to estimate the multidimensionality of wellbeing. We employ factor analysis models and use multivariate EBLUP (MEBLUP) under a unit-level small area estimation approach to predict a vector of means of factor scores representing wellbeing for small areas. We compare this approach to the standard approach whereby we use SAE (univariate and multivariate) to estimate a dashboard of EBLUPs of the means of the original variables and then averaged. Our simulation study shows that the use of factor scores provides estimates with lower variability than weighted and simple averages of standardised MEBLUPs and univariate EBLUPs. Moreover, we find that when the correlation in the observed data is taken into account before small area estimates are computed, multivariate modelling does not provide large improvements in the precision of the estimates over the univariate modelling. We close with an application using the European Union Statistics on Income and Living Conditions data.
\end{abstract}

Keywords: Factor analysis models; Latent variables, Model-based inference; Multivariate EBLUP; Multivariate multilevel models;

\section{Introduction}

The international scientific community, national statistical agencies, and international organisations have pointed out the multidimensional nature of wellbeing as developed under the UN initiative of the Sustainable Development Goals (United Nations, 2017). In particular, government agencies in European Union (EU) countries have been developing wellbeing measurement frameworks. One example is the Italian Statistical Institute (ISTAT) and National Council for Economics and Labour (CNEL) "Equitable and Sustainable Wellbeing (BES)" project (ISTAT and CNEL, 2015). These frameworks generally consist of many dimensions (also called domains), each with many single indicators associated to them. To reduce data dimensionality, summary statistics in the form of a composite indicator may be helpful for policy makers to inform policies targeted towards improving wellbeing. According to OECD-JRC (2008), composite indicators arise when single indicators are compiled into a single index based on an underlying model. The composite indicator measures multidimensional concepts that cannot be studied by single indicators. The set of single indicators estimated individually is referred to as a dashboard of indicators in the social indicators literature. There is on-going debate about the appropriateness of using composite indicators versus a dashboard of single indicators: Ravallion (2011) points out that single multidimensional indicators lead to a loss of information, while Yalonetzky (2012), on the other hand, stresses that composite estimates are necessary when the goal is measuring multiple deprivations (or wellbeing) within the same unit (individual or household). In order to measure multidimensional wellbeing, analysing a dashboard of single indicators (means, totals, ratios, etc.) from the initial set of variables is a standard approach. However, if many indicators need to be analysed, the result may be difficult to interpret. Factor analysis models can be used to reduce data dimensionality and produce composite estimates. In these 
models, the variability among observed correlated variables is described through a smaller number of unobserved latent variables (factors).

In order to inform policy makers who base their decisions on wellbeing measurements, there is a need to obtain accurate and precise indicators at a local area level since wellbeing phenomena are heterogeneous and have different and varying features in territorial areas. However, data obtained from national surveys which measure wellbeing, e.g. income and quality of life, are not reliable at small area levels. One way to overcome this problem is through model-based inference such as small area estimation (SAE) (Rao and Molina, 2015). Small area estimates 'borrow strength' from related small areas through the use of auxiliary variables available at the population level and other related (correlated) dependent variables. As an example, one of the most important social surveys available in EU countries for investigating social phenomena is the Statistics for Income and Living Conditions (EU-SILC). This data can be used to produce accurate direct estimates only at the Nomenclature of Territorial Units for Statistics (NUTS) 2 level (Giusti et al., 2012a) while any areas below this level are unplanned domains with small or even zero sample sizes.

Multivariate SAE, particularly in the unit-level approach, is a research field still under investigation and there is an important gap about social exclusion and wellbeing measurement in a multivariate SAE framework. In the unit-level SAE approach, Fuller and Harter (1987) propose the use of multivariate mixed-effects models in order to predict a vector of means of multiple characteristics of a finite population. Datta et al. (1999) develop a multivariate empirical best linear unbiased predictor (MEBLUP) and empirical bayes (EB) approach for small area mean vectors. They also propose an approximation of the mean squared error and show a gain in efficiency obtainable by using multivariate mixed-effects models compared to univariate models since the correlations between the vector components are taken into account. Molina (2009) deals with the multivariate mixed-effects model under a logarithmic transformation, and Baillo and Molina (2009) study a particular case of the multivariate nested error regression model for uncorrelated random effects. Ngaruye et al. (2017) propose the use of a multivariate linear model for repeated measures data which aims to borrow strength both across small areas and over time. In the area-level SAE approach, Fay (1987) and Datta et al. (1991) consider the multivariate extension to the univariate Fay-Herriot model. Further extensions, applications and estimation procedures are considered in González-Manteiga et al. (2008b), Benavent and Morales (2016) and more recently by Ito and Kubokawa (2018). In this paper, we focus on the unit-level multivariate SAE approach where we assume that the auxiliary variables are known for all units of the sample.

In the classical univariate unit-level SAE approach, the use of the univariate Battese, Harter, and Fuller (BHF) model is widely used (Battese et al., 1988). The model is a mixed-effects model and allows taking into account between-area variability in the prediction stage based on auxiliary information available for the population, such as a register or census. The univariate BHF model can be naturally extended to the multivariate case, where a vector of means becomes the new object of statistical inference.

Moretti et al. (2018b) evaluate the use of factor analysis models in SAE in order to reduce data dimensionality for economic wellbeing indicators in a unit-level univariate SAE approach and show that factor scores can provide good estimates of multidimensional wellbeing phenomena at small area level. A dashboard of single indicators estimated at the small area level using a unit-level univariate 
SAE approach was compared to small area estimates of a single composite indicator arising from the factor analysis model. They showed a gain in terms of the reduction in mean squared error when comparing the estimated mean factor scores with the use of an averaged dashboard of single indicators. According to the factor analysis assumptions, the composite estimates derived from the latent factors are linearly related to the observed variables, and hence have the same economic interpretation. In this paper, we extend Moretti et al. (2018b) by studying the case of more than one latent factor and use a multivariate empirical best linear unbiased predictor (MEBLUP) for factor score mean predictions. This new approach is compared to the averaging of dashboard small area estimates from the original variables using both a univariate and multivariate SAE approach.

In summary, this paper will investigate the following comparisons:

a) Comparison of EBLUP and MEBLUP of single observed response variables;

b) Comparison of EBLUP and MEBLUP of multidimensional latent factors as measured by factor scores;

c) Comparison of the use of latent factors in (b) to a dashboard of single observed response variables expressed as a simple or weighted average of standardised EBLUP and MEBLUP from (a).

The remainder of this paper is organised as follows: in section 2 we introduce the multivariate SAE approach for a mean vector and review the multivariate EBLUP (MEBLUP) under the unit-level multivariate nested-error model. In section 3, we discuss the data dimensionality reduction problem via a factor analysis model. In section 4 , we present a simulation study to evaluate our approach and address the comparisons (a) to (c) above. In section 5, we consider the multidimensionality issue of housing deprivation in Italy through an application using Italian EU-SILC data. We conclude our work in section 6 with a final discussion on the main findings and future research.

\section{Multivariate Empirical Best Linear Unbiased Predictor (MEBLUP)}

Let $d=1, \ldots, D$ denote the small areas for which we want to compute estimates, and let us consider a sample $s \subset \Omega$ of size $n$ drawn from a target finite population $\Omega$ of size $N$. The set of non-sampled units, $N-n$, is denoted by $r$, hence, $s_{d}=s \cap \Omega_{d}$ is the sub-sample from the small area $d$ of size $n_{d}$, $n=\sum_{d=1}^{D} n_{d}$, and $s=\cup_{d} s_{d} \cdot r_{d}$ denotes the set of non-sampled units for small area $d$ of size $N_{d}-$ $n_{d}$

Consider $\boldsymbol{y}_{d i}=\left(\boldsymbol{y}_{d i 1}, \ldots, \boldsymbol{y}_{d i K}\right)$, which denotes the $K$-dimensional row vector of interest on the target $K$ variables for $i=1, \ldots, N_{d}, d=1, \ldots, D$, we can write the target means vector as follows:

$$
\overline{\boldsymbol{y}}_{d}=N_{d}^{-1} \sum_{i=1}^{N_{d}} \boldsymbol{y}_{d i} \text {. }
$$

Hence, because of linearity of this quantity, each area means vector can be split into sampled and non-sampled (out-of-sample) elements as follows:

$$
\overline{\boldsymbol{y}}_{d}=N_{d}^{-1}\left(\sum_{i \in s_{d}} \boldsymbol{y}_{d i}+\sum_{i \in r_{d}} \boldsymbol{y}_{d i}\right) \text {. }
$$

The quantity $\sum_{i \in r_{d}} \boldsymbol{y}_{d i}$ is not observed, so it needs to be predicted. In this work we propose the use of the multivariate mixed-effects model, suggested in SAE by Fuller and Harter (1987) in order to 
predict the out-of-sample observations.

\subsection{Multivariate nested-error linear regression model}

We assume that unit-specific auxiliary variables $\boldsymbol{x}_{d i}$ are available for all the population elements $i$ in each small area $d$ coming from a census or register. We also assume that the following linear model relates the response variables to the auxiliary variables as follows (Fuller and Harter, 1987):

$$
\begin{gathered}
\boldsymbol{y}_{d i}=\boldsymbol{x}_{d i} \boldsymbol{\beta}+\boldsymbol{u}_{d}+\boldsymbol{e}_{d i}, d=1, \ldots, D, i=1, \ldots, N_{d}, \\
\boldsymbol{u}_{d} \stackrel{i i d}{\sim} N_{K}\left(\mathbf{0}, \boldsymbol{\Sigma}_{u}\right), \quad \boldsymbol{e}_{d i} \stackrel{i i d}{\sim} N_{K}\left(\mathbf{0}, \boldsymbol{\Sigma}_{e}\right), \boldsymbol{u}_{d} \text { and } \boldsymbol{e}_{d i} \text { independent }
\end{gathered}
$$

where $\boldsymbol{x}_{d i}$ is a $p$-dimensional row vector of auxiliary variables including the constant 1 as the first term, $\boldsymbol{\beta}$ is a $p \times K$ matrix of unknown regression coefficients, $\boldsymbol{u}_{d}$ is a $K$-dimensional row vector of area effects representing the random variations between small areas not explained by the auxiliary variables, and $\boldsymbol{e}_{d i}$ is $K$-dimensional row vector of the individual effects; $\boldsymbol{u}_{d}$ and $\boldsymbol{e}_{d i}$ are assumed to be independent and normally distributed, $N_{K}$ denotes a $K$-variate Normal distribution. Here, the $K \times$ $K$ positive-definite matrices $\boldsymbol{\Sigma}_{u}$ and $\boldsymbol{\Sigma}_{e}$ are the variance-covariance matrices of the area effects and individual effects, respectively.

In many applications of the multivariate modelling framework, the same covariates are used for all $K$ response variables (Fuller and Harter, 1987; Molina, 2009; Baillo and Molina, 2009; Datta et. al., 1999). For the case of using different covariates for each response variable we refer to other work in multivariate mixed-effects models, such as Goldstein (2011).

\subsection{Estimation and prediction of unknown parameters}

A random sample $s$ of size $n<N$ is drawn from the finite population $\Omega$ according to a sampling design. Model (3) can be written for $i=1, \ldots, n_{d}$ without loss of generality (Rao and Molina, 2015). We make use of the following matrix notation which refers to the sample quantities (Fuller and Harter, 1987):

$\boldsymbol{Y}^{\prime}=\left(\boldsymbol{y}_{11}, \boldsymbol{y}_{12}, \ldots, \boldsymbol{y}_{1, n_{1}}, \ldots, \boldsymbol{y}_{D 1}, \ldots, \boldsymbol{y}_{D, n_{D}}\right)$

$\boldsymbol{X}^{\prime}=\left[\left(\boldsymbol{I}_{K} \otimes \boldsymbol{x}_{11}\right)^{\prime},\left(\boldsymbol{I}_{K} \otimes \boldsymbol{x}_{12}\right)^{\prime}, \ldots,\left(\boldsymbol{I}_{K} \otimes \boldsymbol{x}_{1, n_{1}}\right)^{\prime}, \ldots,\left(\boldsymbol{I}_{K} \otimes \boldsymbol{x}_{D, n_{D}}\right)^{\prime}\right]$

where $\boldsymbol{Y}$ denotes the vector of $n K$ observations on $\boldsymbol{y}_{d i}$ where $\boldsymbol{y}_{d i}$ is defined above, and $\boldsymbol{X}$ denotes the $n K \times p K$ matrix of covariates. The operator $\otimes$ denotes the Kronecker product, and $\boldsymbol{I}$ denotes the identity matrix.

Let us now denote the covariance matrix of $\boldsymbol{Y}$ by

$$
\boldsymbol{V}(\boldsymbol{Y})=\operatorname{block} \operatorname{diag}\left(\boldsymbol{V}_{11}, \ldots, \boldsymbol{V}_{D D}\right)
$$

where $\boldsymbol{V}_{d d}=\left(\boldsymbol{J}_{d d} \otimes \boldsymbol{\Sigma}_{u}\right)+\left(\boldsymbol{I}_{n_{d}} \otimes \boldsymbol{\Sigma}_{e}\right)$. $\boldsymbol{J}_{d d}$ is the $n_{d} \times n_{d}$ matrix with every element equal to one. Let vec $\boldsymbol{\beta}$ denote the column vector of dimension $p K$ obtained by listing the columns of $\boldsymbol{\beta}$ one under the other starting from the first column. The estimator of vec $\boldsymbol{\beta}$ is: 


$$
\operatorname{vec} \widehat{\boldsymbol{\beta}}=\left(\boldsymbol{X}^{\prime} \widehat{\boldsymbol{V}}^{-1} \boldsymbol{X}\right)^{-1} \boldsymbol{X}^{\prime} \widehat{\boldsymbol{V}}^{-1} \boldsymbol{Y}
$$

The empirical best linear unbiased predictors of the random effects are given by (Fuller and Harter, 1987):

$$
\widehat{\boldsymbol{u}}_{d}=\left(\overline{\boldsymbol{y}}_{d, s}-\overline{\boldsymbol{x}}_{d, s} \widehat{\boldsymbol{\beta}}\right)\left[\left(\widehat{\boldsymbol{\Sigma}}_{u}+n_{d}^{-1} \widehat{\boldsymbol{\Sigma}}_{e}\right)^{-1} \widehat{\boldsymbol{\Sigma}}_{u}\right], \quad d=1, \ldots, D
$$

where $\overline{\boldsymbol{y}}_{d, s}$ denotes the sample mean vector and $\overline{\boldsymbol{x}}_{d, s}$ denotes the means of the auxiliary variables in area $d$. The index ' $s$ ' refers to the sample quantities. $\widehat{\boldsymbol{\Sigma}}_{u}$ and $\widehat{\boldsymbol{\Sigma}}_{e}$ are estimators of $\boldsymbol{\Sigma}_{u}$ and $\boldsymbol{\Sigma}_{e}$, respectively. We refer to Schafer et al. (2002) for the estimation algorithm where the maximum likelihood approach is used.

The Multivariate Empirical Best Linear Unbiased Predictor (MEBLUP) of $\overline{\boldsymbol{Y}}_{d}$ is given by (Fuller and Harter, 1987; Rao and Molina, 2015):

$$
\widehat{\overline{\boldsymbol{y}}}_{d}^{M E B L U P}=\overline{\boldsymbol{x}}_{d, p o p} \widehat{\boldsymbol{\beta}}+\widehat{\boldsymbol{u}}_{d}, \quad d=1, \ldots, D,
$$

where $\overline{\boldsymbol{x}}_{d, p o p}$ denotes the known population means of $\boldsymbol{x}_{d i}$ for area $d$. In case of areas with $n_{d}=0$ it holds that $\widehat{\boldsymbol{y}}_{d}^{\text {MEBLUP }}=\widehat{\overline{\boldsymbol{y}}}_{d}^{\text {Syn }}=\overline{\boldsymbol{x}}_{d, p o p} \widehat{\boldsymbol{\beta}}$ (Rao and Molina, 2015); "Syn" denotes the synthetic estimator. We note that for the MEBLUP estimation in formula (7), only the means of the population covariates need to be known $\left(\overline{\boldsymbol{x}}_{d, p o p}\right)$, thus in practise we do not have to link the sample data to the Census data when the sample is small compared to the population (Rao and Molina, 2015; Moretti et al., 2018b).

The mean squared error of (7) can be estimated via resampling techniques, such as the parametric bootstrap, which is widely used in small area estimation under mixed-effects models. We refer to González-Mainteiga et al. (2008a) for statistical theory related to the use of bootstrap to produce MSE estimates under the univariate SAE models. In particular, they show that the parametric bootstrap may provide more accurate MSE estimates compared to analytical approximations due to its secondorder accuracy. Moretti et al. (2018a) extended the parametric bootstrap approach to multivariate SAE and also accounts for $\widehat{\overline{\boldsymbol{y}}}_{d}^{\text {Syn }}$ when $n_{d}=0$ based on the prediction error as in standard linear regression models. In addition, Moretti et al. (2018b) accounts for the error in the factor analysis models in the bootstrap algorithm.

\section{Data dimensionality reduction and the use of factor scores}

Composite indicators are measures for multidimensional phenomena that cannot be studied by the use of single indicators. Due to their complexity, composite indicators should be based on theoretical frameworks and/or definitions to combine single indicators in a way which reflects the phenomena structure (OECD-JRC, 2008). A vast literature on multivariate statistical analysis techniques is available; for a formal review on the main methods we refer to Härdle and Simar (2012). In this paper we assume that latent constructs exist for a wellbeing domain and use factor analysis models to reduce the data dimensionality from the original variables. 


\subsection{The confirmatory factor analysis model}

Let us consider a $K \times 1$ vector of observed random variables $\boldsymbol{Y}$ and we assume that they are linearly dependent on a vector of factors $\boldsymbol{f}$, with dimension $M \times 1(M<K)$. Thus, we can write the following linking model (Kaplan, 2009; Mair, 2018):

$$
\boldsymbol{Y}=\boldsymbol{\Lambda} \boldsymbol{f}+\boldsymbol{\epsilon}
$$

where $\epsilon$ denotes the error associated with the factors (containing both measurement and specific errors), and $\Lambda$ is a $K \times M$ matrix of factor loadings.

Therefore, the implied covariance matrix, also known as the fundamental equation in factor analysis models, is given by (Kaplan, 2009):

$$
\Sigma=\Lambda \Phi \Lambda^{\prime}+\Theta
$$

where $\boldsymbol{\Phi}$ is a $M \times M$ matrix of factor covariance matrix, and $\boldsymbol{\Theta}$ is a $K \times K$ covariance matrix of the errors with $\boldsymbol{\epsilon} \sim N(\mathbf{0}, \boldsymbol{\Theta})$. In confirmatory factor analysis (CFA), restrictions are put on the matrix $\boldsymbol{\Lambda}$ : the elements related to observed variables that are not loaded on a particular factor are fixed to 0 (Kaplan, 2009 and Mair, 2018). The CFA model is also called restricted confirmatory factor analysis model in the literature (see e.g. Kaplan, 2009).

The Maximum Likelihood (ML) approach is used to estimate the model parameters. ML equations under factor analysis models are complicated to solve analytically, so iterative numerical algorithms are proposed in the literature (see Mardia et al., 1979; Jöreskog, 1967; Yang-Wallentin, et al., 2010). Thus, model estimates can be obtained by iteratively minimizing the following function (Kaplan, 2009):

$$
\ell_{M L}=\frac{n}{2}\left[\log |\boldsymbol{\Sigma}|+\operatorname{tr}\left\{\boldsymbol{R} \boldsymbol{\Sigma}^{-\mathbf{1}}\right\}\right]
$$

where $\boldsymbol{R}$ denotes the observed (empirical) covariance matrix.

\subsection{Factor scores estimation in case of continuous observed variables}

After the model parameters are estimated, the factor scores are also estimated. Factor scores are defined as estimates of the values of the unobserved latent variables for each unit $i$. For a review of factor scores estimators we refer to Johnson and Wichern (1998).

Using the regression method, the individual factor scores estimate for sample units are given by (Härdle and Simar, 2012; Lawley and Maxwell, 1971) where $\widehat{\Lambda}$ denotes the estimator of $\boldsymbol{\Lambda}$ :

$$
\widehat{\boldsymbol{f}}_{i}=\widehat{\boldsymbol{\Lambda}}^{\prime} \boldsymbol{R}^{-1} \boldsymbol{y}_{i} .
$$

\subsection{Factor scores estimation when at least one variable in $Y$ is binary or ordered categorical}


In the presence of both binary and continuous observed variables, under a maximum likelihood estimation approach, the factor scores may be estimated via the expected posterior method (Estabrook and Neale, 2013; Boker, et al., 2011; Muthén, 2004) and computed in Mplus 7.4 (Muthén and Muthén, 2012). This estimation procedure is applied in section 5 where an application with real EU-SILC data is proposed.

In the case that at least one variable in $\boldsymbol{Y}$ is binary or ordered categorical then conditional independence is assumed:

$$
g\left(\boldsymbol{y}_{i} \mid \boldsymbol{f}_{i}\right)=\prod_{k=1}^{K} g_{i}\left(y_{i k} \mid \boldsymbol{f}_{i}\right)
$$

here, the factor scores estimates are obtained from the mode of the posterior of $\boldsymbol{f}_{i}$ by minimizing the following function $H$ with respect to $\boldsymbol{f}_{i}$, i.e.:

$$
H=1 / 2\left(\boldsymbol{f}_{i}-\boldsymbol{\mu}_{i}\right)^{\prime} \boldsymbol{\Sigma}^{-1}\left(\boldsymbol{f}_{i}-\boldsymbol{\mu}_{i}\right)-\sum_{k=1}^{K} \ln g\left(\boldsymbol{y}_{i k} \mid \boldsymbol{f}_{i}\right) .
$$

The prior of $\boldsymbol{f}_{\boldsymbol{i}}$ is defined by $\phi\left(\boldsymbol{f}_{\boldsymbol{i}}\right) \sim N\left(\boldsymbol{\mu}_{i}, \boldsymbol{\Sigma}\right)$ and the posterior distribution by $t\left(\boldsymbol{f}_{i} \mid \boldsymbol{y}_{i}\right) \propto$ $\phi\left(\boldsymbol{f}_{\boldsymbol{i}}\right) g\left(\boldsymbol{y}_{i} \mid \boldsymbol{f}_{i}\right)$. The minimization of (13) needs to be done via iterative techniques, such as quasiNewton techniques (Muthén, 2004). Detailed theory related to latent variables modelling in case of non-continuous variables can be found in Muthén (1983) and Muthén (1984).

\section{Simulation study}

This simulation study is designed to assess the feasibility of the multivariate MEBLUP compared to the univariate EBLUP when considering the problem of data dimensionality reduction and the comparisons (a) to (c) mentioned in the introduction.

The overall results of the simulation study are evaluated via the empirical root mean squared error (RMSE) described in Section 4.2.

\subsection{Generating the population}

We generate a single population with $N=20,000, D=80$, and $130 \leq N_{d} \leq 420 . N_{d}$ are generated from the discrete Uniform distribution, $N_{d} \sim d U n i f(130,420)$ with $\sum_{d=1}^{D} N_{d}=20,000 . \boldsymbol{y}_{d i}$ observations are generated according to the multivariate mixed-effects model shown in (3). The simulation parameters $\boldsymbol{\Sigma}_{e}$ and $\boldsymbol{\beta}$ are estimated from real Australian Agricultural and Grazing Industries Survey data (Australia, Bureau of Agricultural Economics, 1978; Molina, 2009). We define the following covariance matrix $\boldsymbol{\Sigma}_{e}$ :

$$
\boldsymbol{\Sigma}_{e}=\left[\begin{array}{cccc}
0.386 & \sigma_{12} & \sigma_{13} & \sigma_{14} \\
\sigma_{21} & 0.414 & \sigma_{23} & \sigma_{24} \\
\sigma_{31} & \sigma_{32} & 0.213 & \sigma_{34} \\
\sigma_{41} & \sigma_{42} & \sigma_{43} & 0.301
\end{array}\right]
$$

Let $r_{u}$ and $r_{e}$ denote the correlation coefficients associated with the covariance matrices $\boldsymbol{\Sigma}_{u}$ and $\boldsymbol{\Sigma}_{e}$ respectively. Hence, $\sigma_{l j}$ with $l \neq j$ in $\Sigma_{e}$ varies according to $r_{e}$. For example, $\sigma_{12}=r_{e} \sqrt{0.386 \cdot 0.414}$ in the above matrix $\boldsymbol{\Sigma}_{e}$. The intra-class correlation coefficients are fixed as follows: $I C C_{k}=$ $\{0.05,0.1,0.3\}$. Therefore the variances of $\boldsymbol{\Sigma}_{u}$ are generated as functions of the variances of $\boldsymbol{\Sigma}_{e}$ as follows: $I C C_{k}=\sigma_{u y_{k}}^{2} /\left(\sigma_{u y_{k}}^{2}+\sigma_{e y_{k}}^{2}\right)$, where $k=1, \ldots, 4$ denote the $k^{\text {th }}$ component of $\boldsymbol{y}_{d i}$. The covariances for $\boldsymbol{\Sigma}_{u}$ are then calculated as described above for $\boldsymbol{\Sigma}_{e}$.

In this simulation we study the following combinations of $r_{u}$ and $r_{e}: r_{u}=r_{e}=0.2, r_{u}=$ 
0.2 and $r_{e}=0.7, r_{u}=-0.2$ and $r_{e}=0.7$.

The $\boldsymbol{\beta}$ regression coefficients matrix (first column relates to the intercept) is given by the following:

$$
\boldsymbol{\beta}=\left[\begin{array}{lll}
1.001 & 0.386 & 0.141 \\
1.187 & 0.377 & 0.133 \\
1.086 & 0.035 & 0.024 \\
0.114 & 0.009 & 0.002
\end{array}\right]
$$

Two uncorrelated covariates are generated from discrete Uniform distributions, $x_{d i 1} \sim d U n i f(145,459), x_{d i 2} \sim d U n i f(55,345)$.

On the generated population, we run two Confirmatory Factor Analysis (CFA) models described in section 3.1: the first model for one latent factor and the second model for two latent factors. This is based on an initial exploratory analysis where we identified that both CFA models provide a good fit to the generated population. We show in Appendix A the goodness of fit statistics of the two CFA models on the generated population for the simulation study. For each latent factor in both CFA models, we estimate the population factor scores from (11), these are denoted by $\boldsymbol{f}_{i}, i=1, \ldots, N$.

Error! Reference source not found. shows how the factors relate to the observed variables for the case of two latent factors in the CFA model.

\section{$<$ Figure 1 about here $>$}

As mentioned in Moretti et al. (2018b), although factor analysis models have been developed to account for multilevel structures, it is not possible to estimate these models for unplanned domains given small and zero sample size domains. Future work will investigate this problem in small area estimation of latent variables.

We also calculate the following true values based on the generated population for each of the small area $d$ : the factor score means, simple averages of the standardized observed variable means, and weighted averages using the CFA loadings denoted by $\bar{Y}_{d m}^{S_{-} \text {Averages }}$ and $\bar{Y}_{d m}^{W_{-} \text {Averages }}$, respectively, where $m$ denotes the $m^{\text {th }}$ factor and the averages are taken over those variables associated to the $m^{\text {th }}$ factor (see Figure 1). The true means are calculated from the generated population to be used in evaluations of the RMSE and BIAS (see formulas (15) and (16)).

For example, the weighted average (based on the factor loadings) of standardized EBLUPs (which have been transformed with zero mean and unit variance) for area $d$ for the variables $k=1, \ldots, K$ that contribute to the $m^{\text {th }}$ factor is given by:

$$
\hat{\bar{Y}}_{d m}^{E B L U P_{-} W_{-} \text {Averages }}=\frac{\sum_{k=1}^{K}\left(\hat{\bar{Y}}_{d k}^{\text {standard_EBLUP }} \widehat{\lambda}_{k m}\right)}{\sum_{k=1}^{K} \widehat{\lambda}_{k m}}, d=1, \ldots, D, m=1, \ldots, M
$$

where $\hat{\lambda}_{k m}$ is the estimated factor loading for variable $k$ related to factor $m$.

\subsection{Simulation steps}

The simulation study consists in the following steps:

1. Draw $S=500$ samples with $n=1,000$ using simple random sampling without replacement from the generated population. The expected sample size per area is $E\left(n_{d}\right)=5$;

2. Fit the one-factor and two-factor CFA model on each sample and estimate the EBLUP factor score means from each model for each area $d$ in each sample. In addition to the separate EBLUP factor score means for each of the factors under the two-factor CFA model, estimate the MEBLUP factor score means; 
3. The EBLUP and MEBLUP for each of the observed variables and vectors $\boldsymbol{Y}$ are also estimated in order to construct simple averages of the standardized small area EBLUPs and MEBLUPs, and a weighted average using the factor loadings estimated in 2 and shown in (14);

4. As the true values are known from the generated population, we can calculate the root mean squared error and the bias for each area $d$ for the different types of estimates: EBLUPs and MEBLUPs of factor score means; and simple and weighted averages of EBLUPs and MEBLUPs. For example, for the univariate EBLUPs of the observed variable mean $k$ denoted by $\hat{\bar{Y}}_{d k}^{E B L U P}$, the root mean squared error is given by:

$$
R M S E\left(\hat{\bar{Y}}_{d k}^{E B L U P}\right)=\sqrt{S^{-1} \sum_{S=1}^{S}\left(\hat{\bar{Y}}_{d k S}^{E B L U P}-\bar{Y}_{d k}^{T R U E}\right)^{2}}
$$

the bias of $\hat{\bar{Y}}_{d k}^{E B L U P}$ is given by:

$$
B I A S\left(\hat{\bar{Y}}_{d k}^{E B L U P}\right)=S^{-1} \sum_{s=1}^{S}\left(\hat{\bar{Y}}_{d k s}^{E B L U P}-\bar{Y}_{d k}^{T R U E}\right)
$$

where $\bar{Y}_{d k}^{T R U E}=N_{d}^{-1} \sum_{i=1}^{N_{d}} y_{i k}$ denotes the true mean of the $Y_{k}$ variable for the $d^{\text {th }}$ area observed in the population.

\subsection{Results of the simulation study}

In this section we describe the main results of the simulation study grouped according to comparisons (a), (b) and (c) as described in the introduction. The Root Mean Squared Error (15) and bias (16) are used as quality measures in order to evaluate the results.

\subsubsection{Comparison (a) of EBLUP and MEBLUP of single observed response variables}

Table 1 shows the percentage relative reduction (in terms of RMSE) of the multivariate MEBLUP over the univariate EBLUP under comparison (a) for single observed response variables. The percentage relative reduction in terms of RMSE for each area is calculated as follows:

$$
\Delta_{d k}=\frac{R M S E\left(\hat{Y}_{d k}^{M E B L U P}\right)-R M S E\left(\hat{Y}_{d k}^{E B L U P}\right)}{R M S E\left(\hat{Y}_{d k}^{E B L U P}\right)} \cdot 100, k=1, \ldots, K, d=1, \ldots, D .
$$

$\Delta_{d k}$ estimates are then averaged across the areas to provide summary statistics for each variable $k$ : $\Delta_{k}=D^{-1} \sum_{d} \Delta_{d k}$

Table 2 presents the bias of the EBLUP and MEBLUP estimates of the observed responses variables averaged over the small area for the three scenarios.

\section{<Table 1 about here> \\ $<$ Table 2 about here $>$}

When the correlations $r_{e}$ and $r_{u}$ are equal to 0.2, we see that the MEBLUP does not provide much improvement over the univariate EBLUP. Indeed, when $r_{e}$ and $r_{u}$ tend to 0 we are close to the independence case, whereby univariate analysis provide the same results as the multivariate analysis (Datta et al., 1999). When correlation coefficients associated to $\boldsymbol{\Sigma}_{e}$ are large, MEBLUP provides more efficient predictions than EBLUP. As it has already been noted by Datta et al. (1999), these gains tend to become large when the signs of $r_{e}$ and $r_{u}$ are opposite. The gains in efficiency are good even when 
the intra-class correlation is low, although we have larger improvements with respect to the RMSE when the intra-class correlation increases. These results confirm previous findings in the multivariate SAE literature, e.g. Datta et al. (1999). Also, although the univariate EBLUPs are all unbiased (very small biases are observed across the small areas), these biases are even smaller in the case of the MEBLUPs. In fact, as pointed out in Berridge and Crouchley (2011), if responses are correlated and we ignore this in the modelling, there is a risk of making errors in the statistical inference. Thus, it is important to consider multivariate EBLUP in case of correlated variables.

\subsubsection{Comparison (b) of EBLUP and MEBLUP of multidimensional latent factors (two-factor CFA model) as measured by factor scores}

Table 3 shows the estimates of the correlation terms between the two factors and the intra-class correlations resulting from the MEBLUP of the two latent factors that were estimated by the twofactor CFA model. It can be seen that the estimated correlation terms and ICC of the two latent factors increase compared to the correlation structure of the original variables when $r_{e}=0.7, r_{u}=0.2$ and $r_{e}=0.7, r_{u}=-0.2$. Under the case $r_{e}=0.2, r_{u}=0.2$ there are mixed results for the correlation term of $r_{u}$ between the two factors and we see a decrease in the estimated ICC.

\section{$<$ Table 3 about here $>$}

Table 4 shows the percentage relative reduction (in terms of RMSE) of the multivariate MEBLUP over the univariate EBLUP of the factor scores. The case $r_{e}=0.2, r_{u}=0.2$ produces smaller ICCs. This means that the MEBLUP has little gain over the univariate EBLUP. The case of $r_{e}=0.7, r_{u}=$ -0.2 and $r_{e}=0.7, r_{u}=0.2$ produce high factor correlations and higher ICCs; thus, increased efficiency of MEBLUP over the EBLUP. Note that the values of the RMSE of the factor scores means SAE predictions are shown in Table 6.

<-Table 4 about here>

\subsubsection{Comparison (c) of the use of latent factors (b) to simple and weighted averages of standardised EBLUP and MEBLUP estimates \\ One-Factor CFA Model}

Table 5 provides the values of the RMSE of the estimates under consideration in comparison (c): simple and weighted averages of standardised original variables for EBLUPs and MEBLUPs and the one-factor CFA factor score means from the univariate SAE EBLUP. Table 6 shows the bias of factor scores means (EBLUP only) from one-factor CFA model, and simple and weighted averages of standardised original variables EBLUP and MEBLUP. Furthermore, Table 7 shows the percentage relative reduction in RMSE for the simple and weighted averages of standardised MEBLUPs over EBLUPs. 
From Table 5, we can see that the RMSEs of the EBLUPs of the factor scores under the one-factor CFA model are all smaller than the RMSEs of the simple and weighted averages of single variables under both the EBLUP and MEBLUP approaches. This confirms findings in Moretti et al. (2018b), which showed that factor score means estimated through EBLUP are more efficient compared to the dashboard approach of taking averages of indicators.

\section{$<$ Table 6 about here $>$}

From Table 6 it can be seen that, although the biases of the estimates coming from the different approaches are all very small, the factor scores produce smaller bias in the estimates compared to simple and weighted averages of standardised EBLUPs and MEBLUPs. This is particularly true when the intra-class correlation is small. When the intra-class correlation is equal to 0.3 the bias reductions in the use of factor scores compared to the averages is not large. The MEBLUP approach provides smaller biases compared to the EBLUP. The bias is generally smaller for the case $r_{e}=0.7, r_{u}=$ -0.2 .

\section{$<$ Table 7 about here $>$}

In addition, the MEBLUP approach for the single variables provides estimates of simple and weighted averages with lower variability than the case where the single variables are estimated under the univariate EBLUP from Table 7. However, we do not see MSE reductions when the correlations in the variance-covariance matrices are small, which is the case when $r_{e}=0.2, r_{u}=0.2$.

\section{Two-Factor CFA Model}

Here we present the results for the two-factor CFA model shown in Figure 1. Table 8 provides the values of the RMSE of each of the estimates under consideration in comparison (c): simple and weighted averages of standardised original variables for EBLUPs and MEBLUPs associated to each of the factors, and the two-factor CFA factor score means from the univariate and multivariate SAE. Table 9 shows the bias of factor score means from two factor CFA model and simple and weighted averages of standardized original variables EBLUP and MEBLUP. Table 10 shows the percentage relative reduction in RMSE for simple and weighted averages of standardised MEBLUPs over EBLUPs for those variables associated to each of the factors in the two-factor CFA model as shown in Table 4.

\section{<Table 8 about here $>$}

Table 8 shows that factor scores produce composite estimates with lower variability than simple and weighted averages for the two-factors case similar to the findings for the one-factor case in Table 5. Also, the MEBLUP provides estimates with lower variability than EBLUP for simple and weighted averages of those variables associated to each of the two factors in the two-factor CFA model. The percentage relative reduction is larger in the case of opposite signs in $r_{e}$ and $r_{u}$. We also see no gains in efficiency when correlations are small.

From Table 9, it can be seen that even in the case of the two-factor CFA model the use of factor scores produce estimates with a smaller bias than the other two approaches. The differences between the bias of the EBLUP and MEBLUP are not always large since they depend on the correlation structure. 


\section{$<$ Table 9 about here $>$}

$<$ Table 10 about here $>$

\subsection{Discussion on simulation study results}

In this simulation study we investigated the use of CFA models in data dimensionality reduction and the application of multivariate SAE for small area indicators. It can be seen that, in line with the general multivariate SAE literature, the use of multivariate mixed-effects models provides estimates with smaller variability than the univariate BHF model when variables are highly correlated with high intra-class correlations. In particular, the percentage of MSE reduction becomes larger when $r_{e}$ and $r_{u}$ have opposite signs. The use of factor score means provide more efficient estimates than the use of the simple and weighted averages of standardised EBLUPs and MEBLUPs of original variables for multidimensional phenomena although they have the same economic interpretation. Interestingly, we can see that if the correlations in the original data are low, we see little or no gain in using an MEBLUP approach compared to the univariate EBLUP. The CFA model produces factor scores to represent latent variables which changes the correlation structures compared to the original variables. In particular, if the intra-class correlation reduces as a result of the CFA model, we see little gain in using the MEBLUP compared to the EBLUP. On the other hand, when correlations in the original data are high, and the correlation structure between factor scores remains high with an increased intraclass correlation, this leads to larger gains in the MEBLUP approach. However, in both cases we see that the MEBLUP approach has less reduction of RSMEs over the univariate EBLUP when considering factor score means estimation, compared to a much larger reduction of RSMEs when comparing simple and weighted averages of small area estimates on the original variables. Thus it appears that when accounting for the correlation structure in the original data a priori through the use of CFA models, we can use a simpler univariate EBLUP approach on each of the factor scores means since there are little gains in using the MEBLUP approach.

\section{Application}

In this section we present an application using real data on housing quality in Italy, focusing on one of the key dimensions in the multidimensional Italian "Economic Wellbeing" of the BES framework. Housing quality is also an important determinant of wellbeing in other Organisation for Economic Co-operation and Development (OECD) countries (Andrews et al., 2011). Data from EU-SILC 2009 and the Italian Census 2001 (for the auxiliary variables) are used. Although the 2009 EU-SILC data were collected in 2008 (seven years after the census), the years 2001-2007 were a period of relatively slow growth and low inflation in Italy (Giusti et al., 2012b). Future work will take into account more recent data for comparisons.

\subsection{Data and variables}

The EU-SILC is conducted yearly by ISTAT for Italy, and coordinated by EUROSTAT at the EU level. For the Italian geography, the survey is designed to produce accurate estimates only at the national and regional levels (NUTS-2) and provinces, whereas municipalities (NUTS-3 and LAU-2 levels), and lower geographical levels are unplanned domains (Giusti et al., 2012a). We use the EUSILC 2009 dataset for Tuscany. The $14^{\text {th }}$ Population and Housing Census 2001 surveyed 1,388,252 households of persons living in Tuscany permanently or temporarily, including the homeless population and persons without a dwelling. Although EU-SILC uses a complex survey design, an 
important feature in the Italian EU-SILC for Tuscany is that every household (and thus adult in the household) has an equal inclusion probability (Eurostat, 2018). Sample designs which implement equal probability selection methods (EPSEM) have many practical advantages and are commonly used in survey practice (Kish, 1995). A sensitivity analysis (not shown here) where the regression models account for the survey design showed no significant differences in the results for the Italian EU-SILC for Tuscany.

We focus on the following sub-dimensions of housing quality (Eurostat, 2016): housing deprivation and problems related to the residential area. Due to data availability, a limited number of variables are selected: severe material deprivation, smog, noise, crime, housing ownership, presence of humidity, darkness inside the house, absence of rubbish in the street, and absence of damages in public buildings. Income is another factor related to wellbeing, although monetary measurement is not always exhaustive for measuring poverty and wellbeing phenomena (Stiglitz et al., 2008). However, income has an interesting effect on housing quality. As Fusco (2015) notes, income and housing deprivation are negatively associated and, in the long run, this relationship becomes stronger. Therefore, it is reasonable to consider income in the analysis of multidimensional housing quality. In our work we use equivalised disposable income denoted by $I^{D E}$, which is calculated as follows (Atkinson et al., 2002):

$$
I_{i}^{D E}=\frac{I_{i}^{D}}{n_{i}^{E}}, i=1, \ldots, N
$$

where $i=1, \ldots, N$ denotes households, $I_{i}^{D}$ is the disposable household income, and $n_{i}^{E}$ is the equivalised household size calculated in the following way:

$$
n_{i}^{E}=1+0.5 \cdot\left(H M_{14+}-1\right)+0.3 \cdot H M_{13-}
$$

where $H M_{14+}$ is the number of household members aged 14 and over at the end of the income reference period, and $H M_{13-}$ is the number of household members aged 13 or younger at the end of the income reference period.

The exploratory variables used in the model (following model-fit diagnostics not shown here) relate to the head of the household and are common to both EU-SILC and Census data. They are gender, age, year of education, household size, size of the flat (in squared metres), and status of employment. Appendix B shows descriptive statistics of the observed variables and auxiliary variables used in the application.

\subsection{Factor analysis and composite estimates}

First, we show results of the unrestricted factor analysis model, also known as Exploratory Factor Analysis (EFA), on the observed variables to investigate their contribution to the total variability (Kaplan, 2009). Table 11 shows the factor structure of the first two factors and how the variables relate to the factors via the factor loadings. According to the factor structure, the following two latent variables can be defined: residential area deprivation (factor 1) and housing material deprivation (factor 2) as shown in Figure 2. Figure 3 shows the scree plot of the EFA eigenvalues where it can be seen that indeed the first two factors explain a good amount of the total variability. Therefore, we keep two factors and carry out the Confirmatory Factor Analysis (CFA) model estimation stage. The factor scores are estimated from the CFA model using Mplus 7.4. For technical aspects on the estimators we refer to Muthén (2004), Muthén (1983) and Muthén (1984). 


\section{$<$ Table 11 about here $>$}

$<$ Figure 2 about here $>$

$<$ Figure 3 about here $>$

The goodness of fit statistics, root mean square error of approximation (RMSEA), the comparative fit index (CFI), and Tucker-Lewis index (TLI) show good results according to Hu and Bentler (1999): $R M S E A=0.040, C F I=0.925$, and $T L I=0.901$. The estimated correlation coefficient between factor 1 and factor 2 is 0.4 . Error! Reference source not found. shows the distributions of the factor scores for each of the latent variables arising from the CFA model following the use of the Box-Cox transformation with a parameter $\delta$ (Box and Cox, 1964) in order to approximate the normal distribution assumption needed for the SAE models. For Factor 1 we used $\delta=3.2$ and for Factor 2 we used $\delta=3.0$.

\section{$<$ Figure 4 about here $>$}

\subsection{Small area estimates and model diagnostics}

Tuscany municipalities are defined as the EU-SILC small areas, with sample sizes ranging from 0 to 135 households. We assume a hierarchical structure in the data with households (level 1) nested within municipalities (level 2). The total number of households in the sample is 1,448 and 59 out of 287 municipalities were sampled. We build two different types of SAE models: first, we apply the univariate BHF approach and consider the factor scores as two separate dependent variables to obtain estimates of the univariate EBLUPs of the single factor means. Also, the multivariate approach is applied and the vector of the factor score means is predicted by MEBLUP. The MSEs of the EBLUPs of factor score means are estimated as in Moretti et al. (2018b). The MSEs of the MEBLUPs are estimated as in Moretti et al. (2018a), taking into account the variability arising from the CFA model as proposed in Moretti et al. (2018b).

In case of areas where $n_{d}=0$ it holds that (Rao and Molina, 2015):

$$
\begin{gathered}
\hat{\bar{f}}_{d m}^{E B L U P}=\hat{\bar{f}}_{d m}^{S y n}=\overline{\boldsymbol{x}}_{d, p o p} \widehat{\boldsymbol{\beta}}^{E B L U P}, m=1,2 \\
\hat{\boldsymbol{f}}_{d}^{M E B L U P}=\hat{\overline{\boldsymbol{f}}}_{d}^{S y n}=\overline{\boldsymbol{x}}_{d, p o p} \widehat{\boldsymbol{\beta}}^{M E B L U P},
\end{gathered}
$$

where $\hat{\bar{f}}_{d m}^{E B L U P}$ and $\hat{\overline{\boldsymbol{f}}}_{d m}^{M E B L U P}$ denote the EBLUP of the mean of the factor scores for the $m^{\text {th }}$ factor and the MEBLUP of the mean vector of factor scores, respectively.

The final EBLUP and MEBLUP factor score means are then transformed for enabling interpretation and mapping using the 'Min-Max' criterion (OECD-JRC, 2008), which transforms the estimates to the interval $[0,1]$. For example, for the EBLUP of the $m=1,2$ factors, the factor scores mean is transformed to a value given by:

$$
\hat{\bar{f}}_{d m}^{E B L U P *}=\frac{\hat{\bar{f}}_{d m}^{E B L U P}-\min \left(\hat{\bar{f}}_{d m}^{E B L U P}\right)}{\max \left(\hat{\bar{f}}_{d m}^{E B L U P}\right)-\min \left(\hat{\bar{f}}_{d m}^{E B L U P}\right)}, \hat{\bar{f}}_{d m}^{E B L U P *} \in[0,1] .
$$

where $\hat{\bar{f}}_{d m}^{E B L U P}$ denotes the EBLUP of factor score means for the $m^{\text {th }}$ factor for small area $d$, the minimum and maximum are across all EBLUPs in areas $d=1, \ldots, D$.

We proceed with the MEBLUP of factor score means and interpret our findings. Table 12 shows the percentiles for the transformed latent housing quality indicators based on MEBLUP of factor score 
means. Error! Reference source not found. shows the maps of residential area deprivation and housing material deprivation, respectively.

\section{$<$ Table 12 about here $>$ \\ $<$ Figure 5 about here $>$}

Although the residential area deprivation dimension is positively correlated with the housing material deprivation dimension, there are important differences at the area level between the two subdimensions. These differences can be seen in the maps. Looking at residential area deprivation estimates (Error! Reference source not found.; left panel) it can be seen that the municipalities located in Massa e Carrara and Siena provinces have the lowest values of the residential area deprivation indicators. Low levels of residential area deprivation are estimated for some municipalities of the south Grosseto province (Manciano and Magliano in Toscana). The highest values in residential area deprivation areas are estimated for municipalities located in the north of the Florence province and north Livorno province. The second map in Error! Reference source not found. (right panel) depicts the housing material deprivation indicator. Interestingly, although the correlation between the two indicators is 0.4 , there are noteworthy differences in some areas: Massa e Carrara, north Siena, Florence, Grosseto and south Siena provinces. For the municipalities located in these provinces the estimates of the housing material deprivation indicator belong to the $4^{\text {th }}$ quantile, denoting high levels of housing material deprivation and belong to the $1^{\text {st }}$ and $2^{\text {nd }}$ quantiles denoting low levels of residential area deprivation.

Error! Reference source not found. and Error! Reference source not found. show the RMSEs of residential area deprivation and housing material deprivation comparing the EBLUP and MEBLUP estimates for those small areas with $n_{d}>0$, respectively.

\section{$<$ Figure 6 about here $>$ \\ $<$ Figure 7 about here $>$}

It can be seen from the figures that the MEBLUP approach provides smaller RMSE over the univariate EBLUP approach. The percentage reduction in terms of RMSE across all areas is $6.41 \%$ and $7.90 \%$ for residential area deprivation and housing material deprivation, respectively.

The model estimates of the variance components and correlations of the latent factors are:

$$
\begin{gathered}
\hat{\sigma}_{e, f_{1}}^{2}=0.086, \sigma_{u, f_{1}}^{2}=0.023, \\
\hat{\sigma}_{e, f_{2}}^{2}=0.170, \sigma_{u, f_{2}}^{2}=0.017, \\
\widehat{\boldsymbol{\Sigma}}_{e}\left[\begin{array}{ll}
0.086 & 0.012 \\
0.012 & 0.169
\end{array}\right], \text { with } \hat{r}_{e}=0.10, \\
\widehat{\boldsymbol{\Sigma}}_{u}\left[\begin{array}{ll}
0.023 & 0.015 \\
0.015 & 0.016
\end{array}\right], \text { with } \hat{r}_{u}=0.78 .
\end{gathered}
$$

The estimated ICCs are 0.21 and 0.09 for factor 1 and factor 2, respectively.

Error! Reference source not found. and Error! Reference source not found. show the Q-Q plots of the residuals (level-1 and level-2) from the univariate BHF and multivariate mixed-effects model, respectively, for both of the factors. It can be seen that the residuals are approximately normally distributed and, in the case of the multivariate mixed-effects model, they behave slightly better. However, it can be noted that level-1 residuals for factor scores 2 behave slightly worse than factor scores 1, particularly in the univariate BHF model. In this case, nonparametric or semi-nonparametric mixed-effects models may be considered. Papageorgiou and Hinde (2010) introduced two families of 
density in these models, the semi-nonparametric and smooth nonparametric densities. Multivariate models with such densities have not been studied in SAE and are a topic for future work. Despite the issues regarding level-1 residuals, our small area estimates are in line with previous studies considering similar economic wellbeing indicators (Moretti, et al., 2018; Marchetti, et al. 2012; Giusti et al. 2015).

Finally, the run-time of the application was negligible and the proposed approach can handle large datasets and number of covariates.

\section{$<$ Figure 8 about here $>$ $<$ Figure 9 about here $>$}

\section{Discussion}

In this paper we evaluated the use of a multivariate empirical best linear unbiased predictor (MEBLUP) under a unit-level mixed-effects model for data dimensionality reduction. In particular, we compared the use of factor score means with the use of simple and weighted averages of standardised EBLUPs and MEBLUPs of original variables in a large-scale simulation study.

The reduction in terms of MSE of the multivariate analysis over the univariate analysis depends on the correlation coefficients $\left(r_{e}\right.$ and $\left.r_{u}\right)$ associated with the variance-covariance matrices and intraclass correlation of the original variables and, in particular, how these change when accounting for the correlations a priori through factor analysis models. This can be seen in the simulation study in comparisons a) and b). Furthermore, when factor score means on several latent variables are used in data dimensionality reduction, these may be estimated using univariate EBLUPs since the correlation structure is accounted for a priori via the factor analysis model. This is shown in the simulation study under comparison (c), where percentages of reduction in terms of RMSE for the factor scores case between MEBLUP and EBLUP are small compared to the reduction in the weighted and simple averages of the original variables. We also show that the standard approach of using a dashboard of indicators, whether calculating each one via univariate BHF model or in a multivariate SAE model have higher RMSE's compared to using factor scores, this is shown in the evaluations of comparison c) of the simulation study.

However, we note that factor scores are still crucial in data dimensionality reduction where different types of variables may arise (binary, continuous, categorical etc.). In fact, in the real data application, we have variables measured on different scales, hence, multivariate EBLUP would require generalised multivariate mixed-effects models, which have not been studied in SAE so far and is a topic for future work. Factor scores estimated by a factor analysis model overcome this issue and allow the study of multidimensional well-being phenomena. In case of skewed distributions, other modelling strategies may be used, such as nonparametric or semi-nonparametric model settings. For example, Papageorgiou and Hinde (2010) considered these families of densities in multivariate generalized mixed-effects models. Attention to these problems in multivariate SAE approaches is a topic for future work.

Funding: This research was financially supported by the United Kingdom Economic and Social Research Council (ESRC) [grant number ES/J500094/1]. 


\section{Appendix A: Goodness of Fit for CFA Models on Generated Population for Simulation Study in Section 4}

Here we present the goodness of fit for the CFA models estimated on the generated population in the simulation study. We consider the following indices: the Standardised Root Mean Square Residual (SRMR), the Comparative Fit Index (CFI) and the Tucker Lewis Index (TLI). SRMR is the square root of the difference between the residuals of the sample covariance matrix and the hypothesised covariance model. It ranges between 0 and 1, and indicates good fit when values equal or lower to 0.08 are assumed (Hu and Bentler, 1999). CFI evaluates the model fit by investigating the discrepancy between the data and the hypothesised model (Gatignon, 2010). Its values range from 0 to 1 , with larger values indicating better fit. A CFI value of 0.95 or higher is accepted as an indicator of good fit (Hu and Bentler, 1999). A TLI range is the same as CFI, for example a TLI equal to 0.95 indicates the considered model improves the fit by $95 \%$ relative to the null model. The cut-off for this index is 0.95 (Hu and Bentler, 1999).

Table A1 Confirmatory factor analysis goodness of fit statistics, one-factor and two-factor model, on the generated population

\begin{tabular}{lccccccc}
\hline & \multicolumn{4}{c}{ One-factor model } & \multicolumn{3}{c}{ Two-factor model } \\
\hline Correlation structure & $I C C_{k}$ & SRMR & CFI & TLI & SRMR & CFI & TLI \\
$\boldsymbol{r}_{\boldsymbol{e}}=\mathbf{0 . 2}, \boldsymbol{r}_{\boldsymbol{u}}=\mathbf{0 . 2}$ & 0.05 & 0.016 & 0.985 & 0.956 & 0.026 & 0.985 & 0.956 \\
& 0.1 & 0.016 & 0.986 & 0.957 & 0.016 & 0.986 & 0.957 \\
& 0.3 & 0.016 & 0.991 & 0.972 & 0.016 & 0.991 & 0.972 \\
$\boldsymbol{r}_{\boldsymbol{e}}=\mathbf{0 . 7}, \boldsymbol{r}_{\boldsymbol{u}}=\mathbf{0 . 2}$ & 0.05 & 0.040 & 0.969 & 0.908 & 0.035 & 0.989 & 0.978 \\
& 0.1 & 0.038 & 0.971 & 0.912 & 0.032 & 0.975 & 0.925 \\
& 0.3 & 0.028 & 0.985 & 0.955 & 0.020 & 0.985 & 0.955 \\
$\boldsymbol{r}_{\boldsymbol{e}}=\mathbf{0 . 7}, \boldsymbol{r}_{\boldsymbol{u}}=-\mathbf{0 . 2}$ & 0.05 & 0.040 & 0.970 & 0.909 & 0.038 & 0.978 & 0.978 \\
& 0.1 & 0.032 & 0.975 & 0.924 & 0.029 & 0.968 & 0.927 \\
& 0.3 & 0.020 & 0.985 & 0.955 & 0.024 & 0.987 & 0.978 \\
\hline
\end{tabular}




\section{Appendix B: Description of variables on EU-SILC 2009 Tuscany data for Application in Section}

5

Table B1 Descriptive statistics of the observed variables (EU-SILC, Tuscany 2009).

\begin{tabular}{lcc}
\hline \multicolumn{1}{c}{ Variable } & Mean & S.D. \\
\hline Severe material deprivation & $4 \%$ & 0.0384 \\
Smog & $17 \%$ & 0.373 \\
Noise & $23 \%$ & 0.424 \\
Crime & $13 \%$ & 0.341 \\
Housing ownership & $74 \%$ & 0.439 \\
Presence of humidity & $15 \%$ & 0.358 \\
Darkness inside the house & $8 \%$ & 0.277 \\
Equivalised disposable income & 20,090 & $13,990.88$ \\
Rooms per household component & 1.989 & 1.239 \\
\hline
\end{tabular}

Table B2 Frequency distribution of access to public services (EU-SILC, Tuscany 2009)

\begin{tabular}{lcc}
\hline \multicolumn{3}{c}{ Access to public services } \\
\hline & Absolute frequency & Relative frequency \% \\
\hline Very difficult & 133 & 9.19 \\
Some difficulties & 249 & 17.20 \\
Easy & 631 & $\mathbf{4 3 . 5 8}$ \\
Very easy & 290 & 20.03 \\
Not needed & 145 & 10.01 \\
\hline Total & 1448 & 100.00
\end{tabular}

Table B3 Frequency distribution of damages to public buildings (EU-SILC, Tuscany 2009)

\begin{tabular}{lcc}
\hline \multicolumn{3}{c}{ Perception of damages to public buildings } \\
\hline & Absolute frequency & Relative frequency $\%$ \\
\hline Always & 65 & 4.49 \\
Often & 83 & 5.73 \\
Sometime & 294 & 20.30 \\
Never & 1006 & $\mathbf{6 9 . 4 8}$ \\
\hline Total & 1448 & 100.00 \\
\hline
\end{tabular}


Table B4 Frequency distribution of perception of rubbish in the street (EU-SILC, Tuscany 2009)

\begin{tabular}{lcc}
\hline & \multicolumn{2}{c}{ Perception of rubbish in the street } \\
\hline & Absolute frequency & Relative frequency $\%$ \\
\hline Always & 75 & 5.18 \\
Often & 82 & 5.66 \\
Sometime & 308 & 21.27 \\
Never & 983 & $\mathbf{6 7 . 8 9}$ \\
\hline Total & 1448 & 100.00 \\
\hline
\end{tabular}

Table B5 Descriptive statistics of the auxiliary variables (EU-SILC, Tuscany 2009)

\begin{tabular}{lcc}
\hline \multicolumn{1}{c}{ Variable } & Mean & S.D. \\
\hline Household size & 2.43 & 1.18 \\
Gender (female) & $70 \%$ & 0.46 \\
Status of employment (employed) & $50 \%$ & 0.50 \\
Age & 57.39 & 16.86 \\
Years of education & 9.76 & 4.56 \\
Flat (or house) size in squared metres & 97.54 & 38.43 \\
\hline
\end{tabular}




\section{Appendix C: Specification of the software used in Section 4 and 5}

Here we describe the main $\mathrm{R}$ packages that can be used to replicate the analysis.

C.1 Estimation of small area means and MSE under univariate EBLUP approach. Although we programmed our functions manually, the sae package (Molina and Marhuenda, 2015) may be used:

- Required packages: nlme, MASS

- Functions: eblupBHF( ) and pbmseBHF( )

nlme and MASS are still required.

C.2 Running Mplus models in the R environment via MplusAutomation (Muthén and Muthén, 2012; Hallquist and Wiley, 2014)

- Functions: mplusObject( ), mplusModeler( )

Mplus is required.

C.3 Mapping using spdep, maptools, sp, Hmisc

- Functions: readShapePoly( ), spplot( ).

C.4 Multivariate mixed-effects model ML fitting via $\mathrm{mlmmm}$ (Yucel, 2010)

- Function: mlmmm.em().

C.5 On the implementation in Mplus

Confirmatory Factor Analysis with continuous and categorical observed variables (application in Section 5)

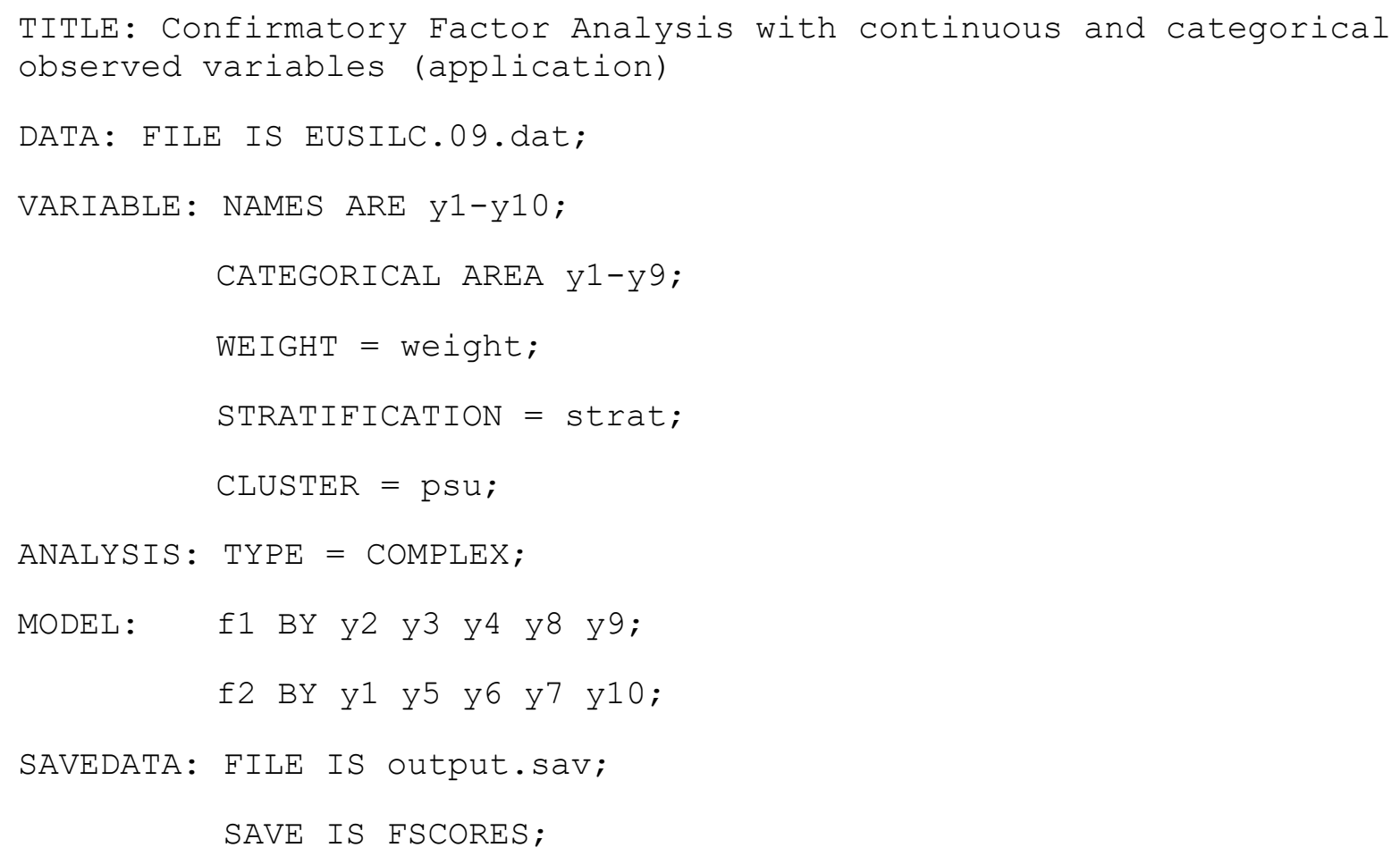

Figure C1 Confirmatory Factor Analysis with continuous and categorical observed variables in Mplus 
Note that the following command, STRATIFICATION $=$ strat, and CLUSTER $=$ psu are used to account for stratification and clustering in the estimation. In our work, after a sensitivity analysis, we decided not to include the commands in the software. We refer to section 5 for more details on the sampling design of the Italian SILC. Theory and more technical aspects on estimators used in this article but under complex sampling designs can be found in Muthén and Satorra (1995). In particular, if weights are included in the analysis, a weighted sample mean vector and weighted sample covariance matrix are used in the estimators". 


\section{References}

Andrews, D., Caldera Sánchez, A., and Johansson, A. (2011) Housing markets and structural policies in OECD countries. OECD Economics Department Working Papers, no 836. OECD Publishing.

Atkinson, T., Cantillon, B, Marlier, E., Nolan, B. (2002) Social Indicators: The EU and Social Inclusion. Oxford University Press, Oxford.

Australia. Bureau of Agricultural Economics (1978), Australian agricultural and grazing industries survey. Grazing industry - sheep and beef cattle industries, Australian Government Publishing Service, Canberra.

Baillo, A. and Molina, I. (2009) Mean Squared Errors of Small-Area Estimators Under a Unit-Level Multivariate Model, Statistics, 43, 553-569.

Bandeed-Roche, K., Miglioretti, D.L., Zeger, S.L. and Rathouz, P.J. (1997) Latent variable regression for multiple discrete outcomes. Journal of the American Statistical Association, 92, 1375-1386.

Battese, G. E., R., Harter, R. M. and Fuller, W. A. (1988) An Error-Components model for Prediction of County crop areas using Survey and Satellite data. Journal of the American Statistical Association, 83 (401) ,28-36.

Benavent, R. and Morales, D. (2016) Multivariate Fay-Herriot models for small area estimation. Computational Statistics \& Data Analysis, 94, 372-390.

Berridge, D.M., and Crouchley, R. (2011) Multivariate Generalized Linear Mixed Models Using R. CRC Press.

Boker S, Neale MC, Maes H, Wilde M, Spiegel M, Brick T, Fox J. (2011) OpenMx: An open source extended structural equation modeling framework. Psychometrika. 76, 306-317.

Box, G. E. P. and Cox, D. R. (1964) An analysis of transformations. Journal of Royal Statistical Society Series B 26, 211-246.

Datta, G. S., Day, B. and Basawa, I. (1999) Empirical best linear unbiased and empirical Bayes prediction in multivariate small area estimation. Journal of Statistical Planning and Inference, 75, 269-279.

Datta, G.S., Fay, R.E., Ghosh, M. (1991) Hierarchical and empirical Bayes multivariate analysis in small area estimation. In: Proceedings of Bureau of the Census 1991 Annual Research Conference, US Bureau of the Census, Washington, DC, 63-79.

Estabrook, R., and Neale, M. (2013) A Comparison of Factor Score Estimation Methods in the Presence of Missing Data: Reliability and an Application to Nicotine Dependence. Multivariate Behavioral Research, 48(1), 1-27.

Eurostat (2018) EU statistics on income and living conditions (EU-SILC) methodology - sampling. Available at: https:/ec.europa.eu/eurostat/statisticsexplained/index.php?title=EU_statistics_on_income_and_living_conditions_(EU-

SILC)_methodology. [Accessed 14 December 2018].

Eurostat (2016) Housing statistics. Retrieved from http://ec.europa.eu/eurostat/statisticsexplained/index.php/Housing_statistics. [Accessed 14 December 2018]

Fay, R. (1987) Application of multivariate regression to small domain estimation, Small Area Statistics in Platek, R. Rao, J.N.K., Sarndal, C.E. and Singh, M.P. (eds), 91-102.

Fuller, W. A. and Harter, R. M. (1987) The Multivariate Components of Variance Model for Small Area Estimation, in R. Platek, J. N. K. Rao, C. E. Sarndal, and M. P. Singh (Eds.), Small Area Statistics, New York: Wiley, 103-123.

Fusco, A. (2015) The relationship between income and housing deprivation: A longitudinal analysis. Economic Modelling, 49, 137-143.

Gatignon, H. (2010) Statistical analysis of management data. Springer. 
Giusti, C., Masserini, L. and Pratesi, M. (2015) Local Comparisons of Small Area Estimates of Poverty: An Application within the Tuscany Region of Italy. Social Indicators Research, 1-20.

Giusti, C., Marchetti, S. and Pratesi, M. (2012a) Estimation of Income Quantiles at the Small Area Level in Tuscany in A. Di Ciaccio et al. (eds.), Advanced Statistical Methods for the Analysis of Large Data-Sets, Studies in Theoretical and Applied Statistics, Springer-Verlag Berlin Heidelberg 2012.

Giusti, C., Marchetti, S., Pratesi, M. and Salvati, N. (2012b) Robust Small Area Estimation and Oversampling in the Estimation of Poverty Indicators. Survey Research Methods, 6(3), 155-163. Goldstein, H. (2011). Multilevel Statistical Models. Fourth edition. Chichester, Wiley.

González-Manteiga, W., Lombardía, M. J., Molina, I., Morales, D. and Santamaría, L. (2008a) Bootstrap Mean Squared Error of a Small-Area EBLUP. Journal of Statistical Computation and Simulation, 78, 443-462.

González-Manteiga, W., Lombardía, M. J., Molina, I., Morales, D. and Santamaría, L. (2008b) Analytic and bootstrap approximations of prediction errors under a multivariate Fay-Herriot model. Computational Statistics \& Data Analysis, 52 (12), 5242-5252

Hallquist, M and Wiley, J. (2014) MplusAutomation: Automating Mplus Model Estimation and Interpretation. $\mathrm{R}$ package version 0.6-3. Retrieved from http://CRAN.Rproject.org/package $=$ MplusAutomation .

Härdle, W. K. and Simar, L. (2012) Applied Multivariate Statistical Analysis. Springer - Verlag Berlin Heidelberg.

Horvitz D.G. and Thompson, D. J. (1952) A Generalization of Sampling without Replacement from Finite Universe. Journal of the American Statistical Association, 47, 663-685.

Hu, L., and Bentler, P. M. (1999) Cutoff criteria for fit indexes in covariance structure analysis: Conventional criteria versus new alternatives. Structural Equation Modeling, 6 (1), 1-55.

ISTAT and CNEL (2015) BES 2015. Il benessere equo e sostenibile in Italia. Report published by ISTAT and CNEL in 2015. from http://www.istat.it/it/files/2015/12/Rapporto_BES 2015.pdf. (In Italian).

Ito and Kubokawa (2018) On Measuring the Variability of Small Area Estimators in a Multivariate Fay-Herriot Model. Available at https://arxiv.org/abs/1804.09941

Johnson, R., Wichern, D. W. (1998) Applied Multivariate Analysis, 4th edition, Prentice.

Jöreskog, K. G. (1969) A general approach to confirmatory maximum likelihood factor analysis. Psychometrika, 34(2), 183-202.

Kaplan, D. (2009) Structural Equation Modeling. Foundations and Extensions. Second Edition. Madison, USA: Sage.

Kish, L. (1995) Survey Sampling. New York: Wiley-Interscience, Revised edition.

Lawley, D.N. and Maxwell, A.E. (1971) Factor Analysis as a Statistical Method. London Butterworths.

Marchetti, S., Tzavidis, N. and Pratesi, M. (2012) Non-parametric bootstrap mean squared error estimation for M-quantile estimators of small area averages, quantiles and poverty indicators, Computational Statistics and Data Analysis, 56, 2889-2902.

Mardia, K. V., Kent, J. T. and Bibby, J. M. (1979) Multivariate Analysis, Academic Press, Duluth, London.

Mair, P. (2018) Modern Psychometrics with R. Springer.

Molina, I. and Marhuenda, Y. (2015) sae: An R Package for Small Area Estimation. The R Journal, 7(1), pp. 81-98. Retrieved from http://journal.r-project.org/archive/2015-1/molinamarhuenda.pdf $>$. 
Molina, I. (2009) Uncertainty under a multivariate nested-error regression model with logarithmic transformation. Journal of Multivariate Analysis, 100, 963-980.

Moretti, A., Shlomo, N., and Sakshaug, J. W. (2018a) Parametric Bootstrap Mean Squared Error of a Small Area Multivariate EBLUP. Communications in Statistics - Simulation and Computation. DOI: $10.1080 / 03610918.2018 .1498889$.

Moretti, A., Shlomo, N., and Sakshaug, J. (2018b) Small Area Estimation of Latent Economic Wellbeing. Sociological Methods \& Research. In press.

Muthén, B.O. (2004) Mplus Technical Appendices. Los Angeles, CA: Muthén and Muthén.

Muthén, B. (1984) A general structural equation model with dichotomous, ordered categorical and continuous latent variable indicators. Psychometrika, 49, 115-132.

Muthén, B. (1983) Latent variable structural equation modelling with categorical data, Journal of Econometrics, 22, 48-65.

Muthén, L.K. and Muthén, B.O. (2012) Mplus User's Guide. Seventh Edition. Los Angeles, CA: Muthén and Muthén.

Muthén, B. and Satorra, A. (1995) Complex sample data in structural equation modelling. In Marsedn, P. V. (Ed.), Sociological Methodology (pp. 267-316). Washington D.C.: The Americal Sociological Association.

Ngaruye, I., Nzabanita, J., von Rosen, D., and Singull, M. (2017) Small area estimation under a multivariate linear model for repeated measures data, Communications in Statistics - Theory and Methods, 46(21), 10835-10850.

OECD-JRC (2008) Handbook on constructing composite indicators: methodology and user guide. OECD Statistics report. Retrieved from http://www.oecd.org/std/42495745.pdf.

Papageorgiou, G., and Hinde, J. (2012) Multivariate generalized linear mixed models with seminonparametric and smooth nonparametric random effects densities. Statistics and Computing, 22, 79-92

Rao, J. N. K. and Molina, I. (2015) Small area estimation. New York: Wiley.

Ravallion, M. (2011) On multidimensional indices of poverty. Journal of Economic Inequality 9(2), 235-248.

Schafer, J. L., and Yucel, R. M. (2002) Computational Strategies for Multivariate Linear MixedEffects Models With Missing Values. Journal of Computational and Graphical Statistics. 11, 437-457.

Stiglitz, J.E., Sen, A., and Fitoussi J.P. (2008) Report by the Commission on the Measurement of Economic Performance and Social Progress, available online at: www.stiglitz-senfitoussi.fr/documents/rapport_anglais.pdf.

United Nations (2017) United Nations sustainable development agenda. Available at http://www.un.org/sustainabledevelopment/development-agenda/.

Yalonetzky, G. (2012) Conditions for the most robust multidimensional poverty comparisons using counting measures and ordinal variables. ECINEQ working paper, 2012-2257.

Yang-Wallentin, F., Jöreskog, K.G. and Luo, H. (2010) Confirmatory Factor Analysis of Ordinal Variables With Misspecified Models. Structural Equation Modeling: A Multidisciplinary Journal. 17(3), 392-423.

Yucel, R. (2010) mlmmm: ML estimation under multivariate linear mixed models with missing values. $\mathrm{R}$ package version 0.3-1.2. Retrieved from http://CRAN.Rproject.org/package $=$ mlmmm. 


\section{Tables in manuscript}

Table 1 Percentage relative reduction (\%) in RMSE of MEBLUP over EBLUP $\left(\Delta_{k}\right)$ for single observed response variables averaged over all areas

\begin{tabular}{ccccc}
\hline \multicolumn{5}{c}{ Scenario } \\
\hline $\boldsymbol{I C} \boldsymbol{C}_{\boldsymbol{k}}$ & & $r_{e}=0.7, r_{u}=0.2$ & $r_{e}=0.7, r_{u}=-0.2$ & $r_{e}=0.2, r_{u}=0.2$ \\
\hline & $\boldsymbol{y}_{\mathbf{1}}$ & -3.50 & -9.21 & -1.04 \\
$\mathbf{0 . 0 5}$ & $\boldsymbol{y}_{\mathbf{2}}$ & -3.00 & -10.81 & -1.02 \\
& $\boldsymbol{y}_{\mathbf{3}}$ & -3.00 & -12.22 & -0.30 \\
& $\boldsymbol{y}_{\mathbf{4}}$ & -2.00 & -12.01 & 0.00 \\
\hline & $\boldsymbol{y}_{\mathbf{1}}$ & -6.00 & -18.42 & -0.31 \\
$\mathbf{0 . 1}$ & $\boldsymbol{y}_{\mathbf{2}}$ & -3.41 & -18.33 & -0.20 \\
& $\boldsymbol{y}_{\mathbf{3}}$ & -6.00 & -19.20 & -0.03 \\
& $\boldsymbol{y}_{\mathbf{4}}$ & -6.02 & -16.90 & -0.09 \\
\hline & $\boldsymbol{y}_{\mathbf{1}}$ & -8.00 & -20.00 & 0.00 \\
$\mathbf{0 . 3}$ & $\boldsymbol{y}_{\mathbf{2}}$ & -7.51 & -19.20 & 0.00 \\
& $\boldsymbol{y}_{\mathbf{3}}$ & -7.03 & -21.11 & 0.00 \\
& $\boldsymbol{y}_{\mathbf{4}}$ & -6.52 & -18.90 & 0.00 \\
\hline
\end{tabular}

Table 2 Bias of EBLUP and MEBLUP for single observed response variables averaged over all areas

\begin{tabular}{|c|c|c|c|c|c|c|c|}
\hline & & & & \multicolumn{4}{|c|}{ Scenario } \\
\hline \multirow[t]{2}{*}{$I C C_{k}$} & & \multicolumn{2}{|c|}{$r_{e}=0.7, r_{u}=0.2$} & \multicolumn{2}{|c|}{$r_{e}=0.7, r_{u}=-0.2$} & \multicolumn{2}{|c|}{$r_{e}=0.2, r_{u}=0.2$} \\
\hline & & $E B L U P$ & $\overline{M E B L U P}$ & $E B L U P$ & $M E B L U P$ & $E B L U P$ & $M E B L U P$ \\
\hline \multirow{4}{*}{0.05} & $y_{1}$ & 0.093 & 0.082 & 0.098 & 0.014 & 0.099 & 0.098 \\
\hline & $y_{2}$ & 0.095 & 0.019 & 0.094 & 0.013 & 0.094 & 0.092 \\
\hline & $y_{3}$ & 0.075 & 0.064 & 0.067 & 0.008 & 0.068 & 0.066 \\
\hline & $y_{4}$ & 0.085 & 0.073 & 0.077 & 0.009 & 0.076 & 0.074 \\
\hline \multirow{4}{*}{0.1} & $y_{1}$ & 0.113 & 0.090 & 0.100 & 0.029 & 0.110 & 0.108 \\
\hline & $y_{2}$ & 0.111 & 0.087 & 0.104 & 0.013 & 0.105 & 0.104 \\
\hline & $y_{3}$ & 0.087 & 0.068 & 0.083 & 0.007 & 0.086 & 0.085 \\
\hline & $y_{4}$ & 0.094 & 0.077 & 0.094 & 0.010 & 0.095 & 0.094 \\
\hline \multirow{4}{*}{0.3} & $y_{1}$ & 0.13 & 0.102 & 0.126 & 0.110 & 0.124 & 0.122 \\
\hline & $y_{2}$ & 0.133 & 0.110 & 0.134 & 0.120 & 0.134 & 0.132 \\
\hline & $y_{3}$ & 0.102 & 0.083 & 0.098 & 0.089 & 0.100 & 0.109 \\
\hline & $y_{4}$ & 0.114 & 0.096 & 0.114 & 0.100 & 0.112 & 0.110 \\
\hline
\end{tabular}


Table $3 \hat{r}_{e}, \hat{r}_{u}$, and ICC of factor scores under multivariate MEBLUP averaged across samples.

\begin{tabular}{|c|c|c|c|c|c|c|c|c|c|}
\hline \multirow[b]{3}{*}{$I C C_{k}$} & \multicolumn{9}{|c|}{ Scenario } \\
\hline & \multicolumn{3}{|c|}{$r_{e}=0.7, r_{u}=0.2$} & \multicolumn{3}{|c|}{$r_{e}=0.7, r_{u}=-0.2$} & \multicolumn{3}{|c|}{$r_{e}=0.2, r_{u}=0.2$} \\
\hline & 0.05 & 0.1 & 0.3 & 0.05 & 0.1 & 0.3 & 0.05 & 0.1 & 0.3 \\
\hline$\hat{\boldsymbol{r}}_{\boldsymbol{e}}$ & 0.85 & 0.70 & 0.60 & 0.75 & 0.70 & 0.62 & 0.53 & 0.63 & 0.75 \\
\hline$\hat{\boldsymbol{r}}_{\boldsymbol{u}}$ & 0.95 & 0.90 & 0.95 & 0.95 & 0.88 & 0.90 & 0.00 & 0.20 & 0.59 \\
\hline$\widehat{I C C} C_{f_{1}}$ & 0.16 & 0.24 & 0.51 & 0.20 & 0.20 & 0.53 & 0.04 & 0.06 & 0.09 \\
\hline$\widehat{I C C} C_{f_{2}}$ & 0.15 & 0.19 & 0.50 & 0.20 & 0.18 & 0.48 & 0.06 & 0.04 & 0.09 \\
\hline
\end{tabular}

Table 4 Percentage relative reduction (\%) in terms of RMSE of MEBLUP of factor scores means over $\operatorname{EBLUP}\left(\Delta_{k}\right)$, two-factor CFA model

\begin{tabular}{ccccc}
\hline \multicolumn{5}{c}{ Scenario } \\
\hline $\boldsymbol{I C}_{\boldsymbol{k}}$ & Factor score & $\boldsymbol{r}_{\boldsymbol{e}}=\mathbf{0 . 7}, \boldsymbol{r}_{\boldsymbol{u}}=\mathbf{0 . 2}$ & $\boldsymbol{r}_{\boldsymbol{e}}=\mathbf{0 . 7}, \boldsymbol{r}_{\boldsymbol{u}}=-\mathbf{0 . 2}$ & $\boldsymbol{r}_{\boldsymbol{e}}=\mathbf{0 . 2}, \boldsymbol{r}_{\boldsymbol{u}}=\mathbf{0 . 2}$ \\
\hline \multirow{2}{*}{$\mathbf{0 . 0 5}$} & Factor 1 & -2.44 & -2.50 & 0.00 \\
& Factor 2 & -2.50 & -2.56 & 0.00 \\
$\mathbf{0 . 1}$ & Factor 1 & -2.56 & -3.13 & 0.00 \\
& Factor 2 & -3.33 & -2.86 & 0.00 \\
$\mathbf{0 . 3}$ & Factor 1 & -4.48 & -5.56 & 0.00 \\
& Factor 2 & -5.56 & -6.67 & 0.00 \\
\hline
\end{tabular}

Table 5 RMSE of factor scores means from one-factor CFA model, and simple and weighted averages of standardised original variables EBLUP/MEBLUP (Bold values highlight smaller RMSE for factor score means under EBLUP).

\begin{tabular}{cccccccc}
\hline \multicolumn{7}{c}{ Scenario } \\
\hline $\boldsymbol{I C}_{\boldsymbol{k}}$ & & $\boldsymbol{r}_{\boldsymbol{e}}=\mathbf{0 . 7}, \boldsymbol{r}_{\boldsymbol{u}}=\mathbf{0 . 2}$ & $\boldsymbol{r}_{\boldsymbol{e}}=\mathbf{0 . 7}, \boldsymbol{r}_{\boldsymbol{u}}=-\mathbf{0 . 2}$ & $\boldsymbol{r}_{\boldsymbol{e}}=\mathbf{0 . 2}, \boldsymbol{r}_{\boldsymbol{u}}=\mathbf{0 . 2}$ \\
\hline & & EBLUP & MEBLUP & EBLUP & MEBLUP & EBLUP & MEBLUP \\
\hline \multirow{3}{*}{$\mathbf{0 . 0 5}$} & Factor scores & $\mathbf{0 . 0 8 1}$ & - & $\mathbf{0 . 0 8 0}$ & - & $\mathbf{0 . 0 7 9}$ & - \\
& Simple averages & 0.267 & 0.244 & 0.231 & 0.181 & 0.230 & 0.228 \\
& Weighted averages & 0.230 & 0.220 & 0.207 & 0.164 & 0.185 & 0.184 \\
\hline \multirow{3}{*}{$\mathbf{0 . 1}$} & Factor scores & $\mathbf{0 . 0 7 0}$ & - & $\mathbf{0 . 0 6 1}$ & - & $\mathbf{0 . 0 6 3}$ & - \\
& Simple averages & 0.246 & 0.225 & 0.250 & 0.180 & 0.207 & 0.205 \\
& Weighted averages & 0.180 & 0.190 & 0.224 & 0.162 & 0.190 & 0.189 \\
\hline \multirow{3}{*}{$\mathbf{0 . 3}$} & Factor scores & $\mathbf{0 . 0 6 5}$ & - & $\mathbf{0 . 0 3 9}$ & - & $\mathbf{0 . 0 7 8}$ & - \\
& Simple averages & 0.200 & 0.177 & 0.181 & 0.160 & 0.198 & 0.197 \\
& Weighted averages & 0.175 & 0.157 & 0.163 & 0.144 & 0.185 & 0.185 \\
\hline
\end{tabular}


Table 6 Bias of factor scores means (EBLUP only) from one-factor CFA model, and simple and weighted averages of standardised original variables EBLUP/MEBLUP

\begin{tabular}{cccccccc}
\hline \multicolumn{7}{c}{ Scenario } \\
\hline $\boldsymbol{I C C}_{\boldsymbol{k}}$ & & $\boldsymbol{r}_{\boldsymbol{e}}=\mathbf{0 . 7}, \boldsymbol{r}_{\boldsymbol{u}}=\mathbf{0 . 2}$ & $\boldsymbol{r}_{\boldsymbol{e}}=\mathbf{0 . 7}, \boldsymbol{r}_{\boldsymbol{u}}=-\mathbf{0 . 2}$ & $\boldsymbol{r}_{\boldsymbol{e}}=\mathbf{0 . 2}, \boldsymbol{r}_{\boldsymbol{u}}=\mathbf{0 . 2}$ \\
\hline & EBLUP & MEBLUP & EBLUP & MEBLUP & EBLUP & MEBLUP \\
\hline \multirow{3}{*}{$\mathbf{0 . 0 5}$} & Factor scores & $\mathbf{0 . 0 0 5}$ & - & $\mathbf{0 . 0 0 4}$ & - & $\mathbf{0 . 0 0 6}$ & - \\
& Simple averages & 0.014 & 0.010 & 0.008 & 0.007 & 0.010 & 0.009 \\
& Weighted averages & 0.008 & 0.006 & 0.007 & 0.006 & 0.008 & 0.008 \\
\hline \multirow{3}{*}{$\mathbf{0 . 1}$} & Factor scores & $\mathbf{0 . 0 0 1}$ & - & $\mathbf{0 . 0 0 1}$ & - & $\mathbf{0 . 0 0 0}$ & - \\
& Simple averages & 0.012 & 0.010 & 0.003 & 0.001 & 0.001 & 0.001 \\
& Weighted averages & 0.005 & 0.003 & 0.003 & 0.001 & 0.001 & 0.001 \\
\hline \multirow{3}{*}{$\mathbf{0 . 3}$} & Factor scores & $\mathbf{0 . 0 0 1}$ & - & $\mathbf{0 . 0 0 1}$ & - & $\mathbf{0 . 0 0 1}$ & - \\
& Simple averages & 0.004 & 0.003 & 0.004 & 0.003 & 0.002 & 0.002 \\
& Weighted averages & 0.003 & 0.002 & 0.003 & 0.002 & 0.001 & 0.001 \\
\hline
\end{tabular}

Table 7 Percentage relative reduction (\%) in terms of RMSE of simple and weighted averages of standardised MEBLUP over EBLUP $\left(\Delta_{k}\right)$.

\begin{tabular}{ccccc}
\hline \multicolumn{5}{c}{ Scenario } \\
\hline $\boldsymbol{I C C}_{\boldsymbol{k}}$ & & $\boldsymbol{r}_{\boldsymbol{e}}=\mathbf{0 . 7}, \boldsymbol{r}_{\boldsymbol{u}}=\mathbf{0 . 2}$ & $\boldsymbol{r}_{\boldsymbol{e}}=\mathbf{0 . 7}, \boldsymbol{r}_{\boldsymbol{u}}=-\mathbf{0 . 2}$ & $\boldsymbol{r}_{\boldsymbol{e}}=\mathbf{0 . 2}, \boldsymbol{r}_{\boldsymbol{u}}=\mathbf{0 . 2}$ \\
\hline \multirow{2}{*}{$\mathbf{0 . 0 5}$} & Simple averages & -8.61 & -21.65 & -0.87 \\
& Weighted averages & -4.35 & -20.77 & -0.54 \\
\hline \multirow{2}{*}{$\mathbf{0 . 1}$} & Simple averages & -8.54 & -28.00 & 0.00 \\
& Weighted averages & -5.56 & -27.68 & 0.00 \\
\hline \multirow{2}{*}{$\mathbf{0 . 3}$} & Simple averages & -11.50 & -11.60 & -0.51 \\
& Weighted averages & -10.29 & -11.66 & 0.00 \\
\hline
\end{tabular}


Table 8 RMSE of factor score means from two factor CFA model and simple and weighted averages of standardized original variables EBLUP/MEBLUP (Bold values highlight smaller RMSE for factor score means under EBLUP/MEBLUP).

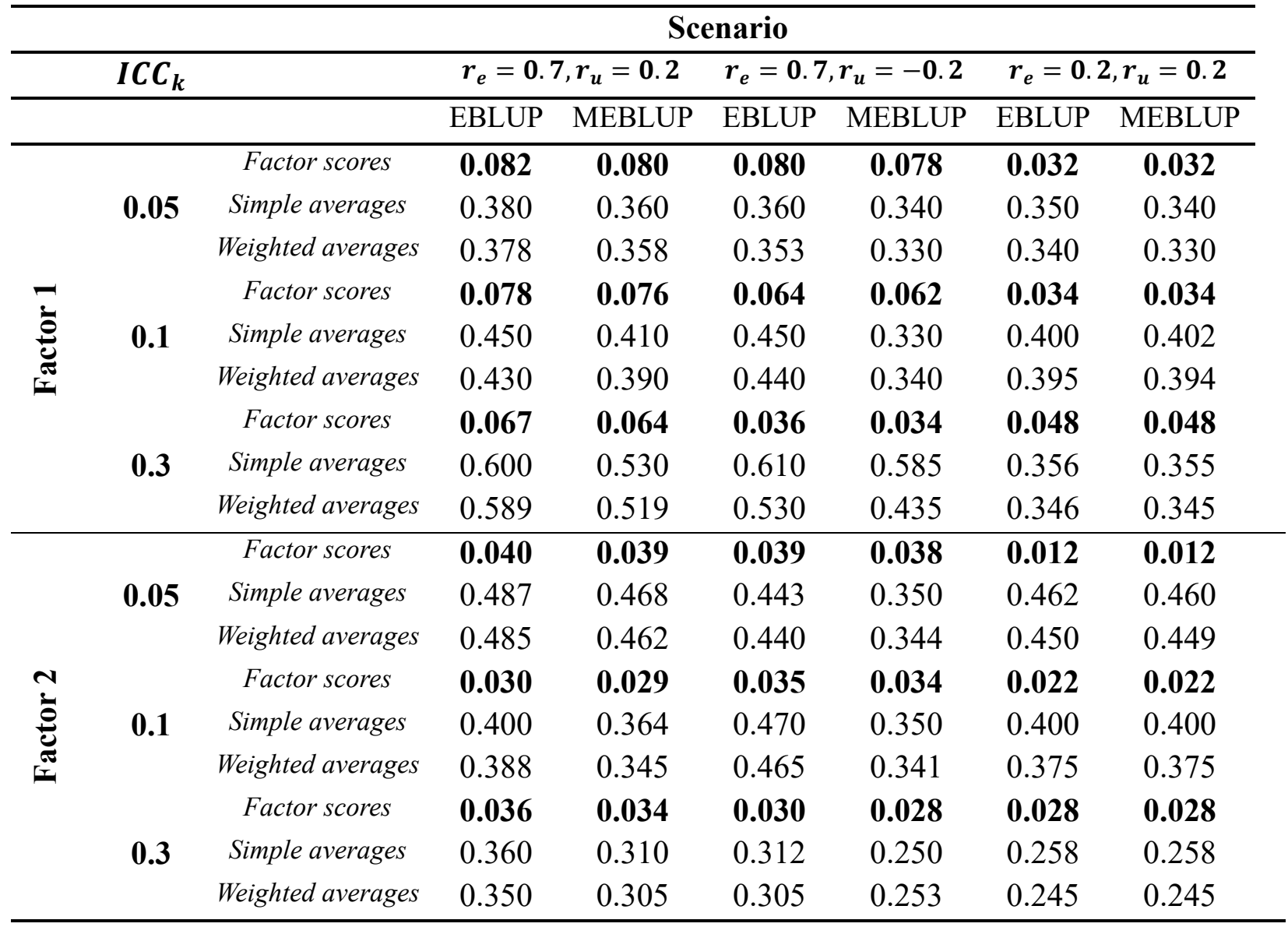


Table 9 Bias of factor score means from two factor CFA model and simple and weighted averages of standardized original variables EBLUP/MEBLUP

\begin{tabular}{|c|c|c|c|c|c|c|c|c|}
\hline \multirow{2}{*}{\multicolumn{2}{|c|}{$I C C_{k}$}} & \multicolumn{7}{|c|}{ Scenario } \\
\hline & & & \multicolumn{2}{|c|}{$r_{e}=0.7, r_{u}=0.2$} & \multicolumn{2}{|c|}{$r_{e}=0.7, r_{u}=-0.2$} & \multicolumn{2}{|c|}{$r_{e}=0.2, r_{u}=0.2$} \\
\hline & & & EBLU & MEBLU & EBLU & MEBLU & EBLU & MEBLU \\
\hline & & & $\mathrm{P}$ & $\mathrm{P}$ & $\mathrm{P}$ & $\mathrm{P}$ & $\mathrm{P}$ & $\mathrm{P}$ \\
\hline \multirow{9}{*}{ لَّ } & \multirow{3}{*}{0.05} & Factor scores & 0.067 & 0.065 & 0.014 & 0.010 & 0.027 & 0.025 \\
\hline & & Simple averages & 0.082 & 0.032 & 0.029 & 0.015 & 0.031 & 0.025 \\
\hline & & $\begin{array}{l}\text { Weighted } \\
\text { averages }\end{array}$ & 0.080 & 0.029 & 0.020 & 0.012 & 0.029 & 0.028 \\
\hline & \multirow{3}{*}{0.1} & Factor scores & 0.063 & 0.061 & 0.025 & 0.015 & 0.028 & 0.027 \\
\hline & & Simple averages & 0.083 & 0.075 & 0.033 & 0.020 & 0.034 & 0.034 \\
\hline & & $\begin{array}{l}\text { Weighted } \\
\text { averages }\end{array}$ & 0.080 & 0.071 & 0.029 & 0.020 & 0.031 & 0.031 \\
\hline & \multirow{3}{*}{0.3} & Factor scores & 0.053 & 0.052 & 0.029 & 0.028 & 0.030 & 0.028 \\
\hline & & Simple averages & 0.060 & 0.059 & 0.036 & 0.035 & 0.039 & 0.032 \\
\hline & & $\begin{array}{l}\text { Weighted } \\
\text { averages }\end{array}$ & 0.058 & 0.058 & 0.030 & 0.029 & 0.037 & 0.031 \\
\hline \multirow{9}{*}{ 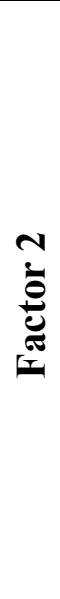 } & \multirow{3}{*}{0.05} & Factor scores & 0.031 & 0.030 & 0.031 & 0.030 & 0.017 & 0.017 \\
\hline & & Simple averages & 0.384 & 0.383 & 0.403 & 0.390 & 0.399 & 0.399 \\
\hline & & $\begin{array}{l}\text { Weighted } \\
\text { averages }\end{array}$ & 0.380 & 0.378 & 0.399 & 0.388 & 0.398 & 0.397 \\
\hline & \multirow{3}{*}{0.1} & Factor scores & 0.030 & 0.026 & 0.030 & 0.030 & 0.018 & 0.017 \\
\hline & & Simple averages & 0.320 & 0.320 & 0.399 & 0.380 & 0.320 & 0.320 \\
\hline & & $\begin{array}{l}\text { Weighted } \\
\text { averages }\end{array}$ & 0.318 & 0.317 & 0.395 & 0.375 & 0.318 & 0.317 \\
\hline & \multirow{3}{*}{0.3} & Factor scores & 0.024 & 0.022 & 0.028 & 0.027 & 0.033 & 0.032 \\
\hline & & Simple averages & 0.350 & 0.349 & 0.283 & 0.255 & 0.040 & 0.039 \\
\hline & & $\begin{array}{l}\text { Weighted } \\
\text { averages }\end{array}$ & 0.348 & 0.340 & 0.280 & 0.250 & 0.038 & 0.037 \\
\hline
\end{tabular}

Table 10 Percentage relative reduction (\%) in terms of RMSE for simple and weighted averages of variables associated to each of the factors of MEBLUP over EBLUP, $\left(\Delta_{k}\right)$ two-factors CFA model.

\begin{tabular}{cccccccc}
\hline \multicolumn{8}{c}{ Scenario } \\
\hline $\boldsymbol{I C C}_{\boldsymbol{k}}$ & \multicolumn{5}{c}{$\boldsymbol{r}_{\boldsymbol{e}}=\mathbf{0 . 7}, \boldsymbol{r}_{\boldsymbol{u}}=\mathbf{0 . 2}$} & $\boldsymbol{r}_{\boldsymbol{e}}=\mathbf{0 . 7}, \boldsymbol{r}_{\boldsymbol{u}}=-\mathbf{0 . 2}$ & $\boldsymbol{r}_{\boldsymbol{e}}=\mathbf{0 . 2}, \boldsymbol{r}_{\boldsymbol{u}}=\mathbf{0 . 2}$ \\
\hline $\mathbf{0 . 0 5}$ & Factor 1 & -5.26 & -5.29 & -5.56 & -6.52 & -2.86 & -2.94 \\
& Factor 2 & -3.90 & -4.74 & -20.99 & -21.82 & -0.43 & -0.22 \\
$\mathbf{0 . 1}$ & Factor 1 & -8.89 & -9.30 & -26.67 & -22.73 & -0.50 & -0.25 \\
& Factor 2 & -9.00 & -11.08 & -25.53 & -26.67 & 0.00 & 0.00 \\
$\mathbf{0 . 3}$ & Factor 1 & -11.67 & -11.88 & -16.67 & -17.92 & -0.28 & -0.29 \\
& Factor 2 & -13.89 & -12.86 & -19.87 & -17.05 & 0.00 & 0.00 \\
\hline
\end{tabular}


Table 11 Factor structure for two latent factors using EFA.

\begin{tabular}{lccc}
\hline \multicolumn{1}{c}{ Variable } & & Factor & Factor 2 \\
& \multicolumn{1}{c}{$\mathbf{1}$} \\
\hline Severe material deprivation & $y_{1}$ & 0.010 & $\mathbf{0 . 7 3 3}$ \\
Smog & $y_{2}$ & $\mathbf{0 . 7 5 7}$ & 0.025 \\
Noise & $y_{3}$ & $\mathbf{0 . 6 1 7}$ & 0.154 \\
Crime & $y_{4}$ & $\mathbf{0 . 6 5 9}$ & 0.130 \\
Housing ownership & $y_{5}$ & 0.096 & $\mathbf{- 0 . 5 8 9}$ \\
Presence of humidity & $y_{6}$ & 0.010 & $\mathbf{0 . 5 9 6}$ \\
Darkness inside the house & $y_{7}$ & -0.002 & $\mathbf{0 . 5 5 1}$ \\
Absence of rubbish in the street & $y_{8}$ & $\mathbf{- 0 . 8 4 3}$ & 0.084 \\
Absence of damages in public buildings & $y_{9}$ & $\mathbf{- 0 . 8 1 0}$ & 0.012 \\
Log equivalised disposable income & $y_{10}$ & 0.139 & $\mathbf{- 0 . 3 9 8}$ \\
\hline
\end{tabular}

Table 12 Percentiles for transformed latent housing quality indicators based on MEBLUP of factor score means.

\begin{tabular}{lccccc}
\hline & \multicolumn{5}{c}{ MEBLUP Percentile } \\
\cline { 2 - 6 } & $0 \%$ & $25 \%$ & $50 \%$ & $75 \%$ & $100 \%$ \\
\hline Residential area deprivation & 0.000 & 0.261 & 0.266 & 0.270 & 1.000 \\
Housing material deprivation & 0.000 & 0.418 & 0.457 & 0.502 & 1.000
\end{tabular}

